Research Article

\title{
Modeling and Vibration Analysis of a Porous Rotational Shell Based on Biot Theory
}

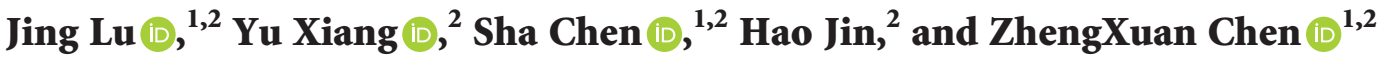 \\ ${ }^{1}$ School of Mechanical and Transportation Engineering, Guangxi University of Science and Technology, Liuzhou 545006, China \\ ${ }^{2}$ Guangxi Key Laboratory of Automobile Components and Vehicle Technology, Guangxi University of Science and Technology, \\ Liuzhou 545006, China
}

Correspondence should be addressed to Yu Xiang; gxutxly@126.com

Received 4 November 2020; Revised 2 December 2020; Accepted 17 December 2020; Published 13 January 2021

Academic Editor: Jie Yang

Copyright (C) 2021 Jing Lu et al. This is an open access article distributed under the Creative Commons Attribution License, which permits unrestricted use, distribution, and reproduction in any medium, provided the original work is properly cited.

Combining the Biot theory and classical elastic theory for thin shells, a new dynamic model of a thin fluid-saturated porous rotational shell is proposed. First-order ordinary differential control equations of the porous rotational shell are derived in the frequency domain. These equations are then solved by using the precise element method. The accuracy of this model has been verified by comparing with a vibration experiment. Moreover, the comparisons between the present model and two equivalent property models are carried out. Because the present approach considers the fluid-solid coupling effect and makes no assumptions for the fluid displacements, it is more accurate in the high-frequency range. Lastly, the dynamic characteristics of porous rotational shells are demonstrated by the proposed method.

\section{Introduction}

Porous materials are widely used for passive absorption and noise control in many fields, such as the vehicle, aerospace, geophysics, and civil engineering. Many researchers have studied the vibration and acoustic characteristic of poroelastic structures since the 1950s. In 1956, Biot [1, 2] established the three-dimensional theory of wave propagation in fluid-saturated porous elastic solids to describe the linear acoustic behaviours of these porous media. Several representative formulations are presented by Zienkiewicz et al. [3, 4], such as $u-U-p, u-U, u-p$, and $u$ - $w$ forms. Recently, Cieszko and Kubik [5] put forward the analysis of equations to describe wave propagation in fluid-saturated porous materials. By applying some assumptions, the Biot theory has been applied to the research on porous plates and shells. For instance, neglecting the relative fluid/solid displacements and defining the in-plane solid displacements as functions of the transverse solid displacement, Manuel et al. derived a mixed displacement-pressure formulation for the bending vibration of porous plates by combining the thin plate theory and Biot theory [6]. This formulation is useful for porous plates saturated by air. In order to evaluate the energy dissipation due to the viscous friction, Leclaire and Horoshenkov proposed an analytical model of a fluid-saturated porous plate by considering the fluid loading terms and introducing relative transverse fluid/solid displacements [7]. However, it was pointed out that the model was only valid for low frequencies since the thickness of the plate was assumed to be smaller than the acoustic wavelength and the variation of the amplitude of the transverse fluid displacement was also assumed to be small. Xiang et al. [8] proposed a precise dynamic model of a thin rectangular porous plate based on the classical theory of thin plate. Theodorakopoulos and Beskos [9] assumed that the in-plane fluid flow relative to the motion of the solid was negligible compared to the 
transverse fluid flow, and the governing equation written in terms of the three solid displacements and fluid transverse displacement was derived to demonstrate the flexural plate dynamic characteristic in the frequency domain. Based on a similar hypothesis, research on porous cylindrical shells has been carried out. Ahmed Shah et al. obtained a frequency domain equation for the axially symmetric vibration of an infinite poroelastic circular cylindrical shell with different boundary conditions [10]. Employing a first-order shear deformation theory and the 3D Biot theory, Julien et al. derived an analytical model of an infinite sandwich cylindrical shell to demonstrate the influence of the structural damping [11]. However, these infinite cylindrical shell models are quite different from the shells used in practical applications. Due to the difficulty of analytical modeling of this problem, many numerical methods were adopted $[12,13]$, and the finite element method (FEM) combined with equivalent models is frequently implemented instead of the analytical method $[14,15]$. Boily and Charron created a finite element model of a sandwich cylindrical shell [16]. In the model, the poroelastic core was simplified to an equivalent fluid model, and the modal reduction method was used to reduce the size of the system. The finite element method is convenient and widely used, but the FEM model of porous structure is usually large, complex, and timeconsuming, especially at high frequencies. Therefore, this method can only be practically applied in low-frequency cases. On the other hand, rotational shells are widely used in various fields, such as aerospace, ships, and civil machinery. Porous is usually treated on these rotational shells to reduce the noise and isolate the vibration. However, the existing research studies mostly focus on porous beams, plates, and cylindrical shells, and vibration analyses of rotational porous shells are not available in the literature.

In this article, accounting for the coupling interaction between the fluid and solid phases, the dynamic governing equations of the porous rotational shell based on classical thin shell theory and Biot theory are derived. The equation is written in a first-order ordinary differential matrix form and solved using a precise integration method. Subsequently, corresponding experiment results are used to verify the accuracy of the present method. Finally, some additional numerical examples are demonstrated.

\section{Governing Equations}

2.1. Constitutive Equations of the Porous Rotational Shell. The stress-strain relations of a homogeneous, isotropic poroelastic material are $[1,2]$

$$
\begin{aligned}
\sigma_{i j} & =2 N e_{i j}+(A e+Q \varepsilon) \delta_{i j}, \quad(i, j=1,2,3), \\
-\phi p & =Q e+R \varepsilon .
\end{aligned}
$$

The first set of formulas in equation (1) describes the constitutive relations of the solid phase, and the second set of formulas describes the constitutive relations of the fluid phase. The elastic coefficients of the porous material $A$ and $N$ have a similar meaning with the Lamé coefficients for an elastic material. $N$ is the shear modulus of the skeleton material. $R$ is a pressure coefficient forcing a certain volume of the fluid into a constant aggregate. $Q$ represents the coupling coefficient between the volume change of the solid and liquid. $\delta_{i j}$ is the Kronecker delta. $p$ is the fluid pressure. $\phi$ is the porosity. $\sigma_{i j}$ and $e_{i j}$ are the stress and strain tensor of the solid skeleton, respectively. $e$ and $\varepsilon$ are the dilations of solid and fluid, respectively, which are given by

$$
\begin{aligned}
& e=e_{k k}=u_{k, k}, \\
& \varepsilon=\varepsilon_{k k}=U_{k, k}, \quad k=1,2,3,
\end{aligned}
$$

where $u$ is the solid displacement tensor and $U$ is the fluid displacement tensor. Note that the repetition of the index $k$ implies summation; commas indicate differentiation with respect to space variables; and $\varepsilon_{i j}$ denotes the fluid strain tensor. In a spherical coordinate system, $k$ is equal to $\varphi, \theta$, or $z$.

The middle plane of the thin porous rotational shell coincides with the natural coordinates, as shown in Figure 1, in which, $\varphi, \theta$, and $z$ are meridian, circumferential, and normal coordinates, respectively, with relevant curvature radii of $R_{\varphi}$ and $R_{\theta}$, respectively. The midplane displacements in the meridian, circumferential, and normal directions are $u, v$, and $w$, respectively. The midplane Lamé coefficients in meridian and circumferential directions are $R_{\varphi}$ and $R_{0}$, respectively, and are both the functions of $\varphi$.

Applying the classical elastic theory for a thin shell, the normal line ( $z$-direction) of the middle plane is assumed to be straight. Additionally, the angles between the normal line and the vertical lines of the meridian and circumferential direction are constants. Furthermore, the total normal stress can be neglected. Applying these assumptions, one obtains

$$
\begin{aligned}
e_{\varphi z} & =0, \\
e_{\theta z} & =0, \\
\sigma_{z}-\phi p \eta & =0 .
\end{aligned}
$$

The fluid dilation can be evaluated from the second set of formulas in equation (1) as

$$
\varepsilon=\frac{-\phi p-Q e}{R} \text {. }
$$

Substituting equation (4) into the first set of formulas of equation (1) and omitting the two shear stress components according to equation (3) yield 


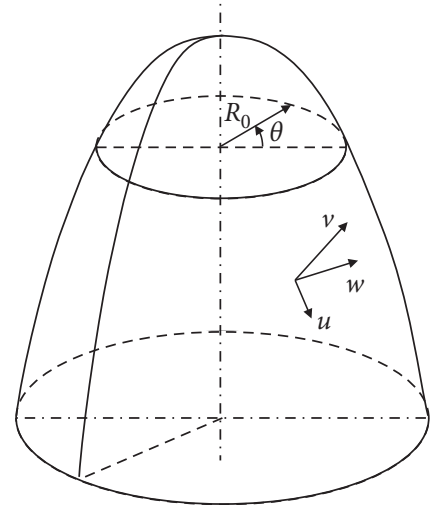

Figure 1: The porous rotational shell.

$$
\begin{aligned}
\sigma_{\varphi} & =2 N e_{\varphi}+\left(A-\frac{Q^{2}}{R}\right) e-\frac{Q}{R} \phi p, \\
\sigma_{\theta} & =2 N e_{\theta}+\left(A-\frac{Q^{2}}{R}\right) e-\frac{Q}{R} \phi p, \\
\sigma_{z} & =2 N e_{z}+\left(A-\frac{Q^{2}}{R}\right) e-\frac{Q}{R} \phi p, \\
\tau_{\varphi \theta} & =N e_{\varphi \theta} .
\end{aligned}
$$

By eliminating $e_{z}$ from the third formula of equation (5) with the third formula of equation (3), the solid dilation is obtained:

$$
e=(1-\bar{B})\left(e_{\phi}+e_{\theta}\right)+\bar{A} \phi p,
$$

where $\bar{A}$ and $\bar{B}$ are constants, with $\bar{A}=((Q+R) /$ $\left.\left(2 N R+A R-Q^{2}\right)\right), \bar{B}=\left(\left(A R-Q^{2}\right) /\left(2 N R+A R-Q^{2}\right)\right)$.

Combing equations (5) and (6), the solid constitutive equations of a porous rotational shell take the following form:

$$
\begin{aligned}
\sigma_{\varphi} & =\overline{\bar{N}} e_{\varphi}+\overline{\bar{A}} e_{\theta}+\overline{\bar{B}} \phi p, \\
\sigma_{\theta} & =\overline{\bar{N}} e_{\theta}+\overline{\bar{A}} e_{\varphi}+\overline{\bar{B}} \phi p, \\
\tau_{\varphi \theta} & =N e_{\varphi \theta},
\end{aligned}
$$

where $\overline{\bar{A}}=\left(A-\left(Q^{2} / R\right)\right)(1-\bar{B}), \overline{\bar{B}}=\left[\left(A-\left(Q^{2} / R\right)\right) \bar{A}-\left(Q^{2}\right.\right.$ $/ R)], \overline{\bar{N}}=\left[2 N+\left(A-\left(Q^{2} / R\right)\right)(1-\bar{B})\right]$.

Substituting equation (6) into equation (4), the fluid constitutive equations of a porous rotational shell are obtained:

$$
\varepsilon=\overline{\bar{A}}^{\prime} \phi p+\overline{\bar{B}}^{\prime}\left(e_{\varphi}+e_{\theta}\right),
$$

where $\overline{\bar{A}}^{\prime}=-((1+Q \bar{A}) / R), \overline{\bar{B}}^{\prime}=-(Q / R)(1-\bar{B})$.

2.2. Governing Equations of the Solid Frame. Applying the Reissner model with classical thin shell theory, the solid displacements can be written as [17]

$$
\begin{aligned}
& \widehat{u}_{s}=u_{s}+z \theta_{\varphi}, \\
& \widehat{v}_{s}=v_{s}+\frac{z}{R_{0}}\left(v_{s} \sin \varphi-\frac{\partial w_{s}}{\partial \theta}\right), \\
& \widehat{w}_{s}=w_{s},
\end{aligned}
$$

where $\widehat{u}_{s}=\widehat{u}_{1}, \widehat{v}_{s}=\widehat{u}_{2}, \widehat{w}_{s}=\widehat{u}_{3}$ are the solid displacements at an arbitrary point of the shell. The displacements $u_{s}, v_{s}, w_{s}$ correspond to the midplane displacements. The midplane rotation $\theta_{\varphi}$ is

$$
\theta_{\varphi}=\frac{1}{R_{\varphi}}\left(u_{s}-\frac{\partial w_{s}}{\partial \varphi}\right)
$$

The corresponding solid strains can be represented using the midplane solid displacements as follows [17]:

$$
\left\{\begin{array}{l}
e_{\varphi}=\left(\frac{1}{R_{\varphi}}\right) \frac{\partial u_{s}}{\partial \varphi}+\left(\frac{1}{R_{\varphi}}\right) w_{s}+\frac{z}{R_{\varphi}} \frac{\partial \theta_{\varphi}}{\partial \varphi} \\
e_{\theta}=\left(\frac{\cos \varphi}{R_{0}}\right) u_{s}+\left(\frac{1}{R_{0}}\right) \frac{\partial v_{s}}{\partial \theta}+\left(\frac{\sin \varphi}{R_{0}}\right) w_{s}+\frac{z}{R_{0}} \frac{\partial}{\partial \theta}\left(\frac{\sin \varphi}{R_{0}} v_{s}-\frac{1}{R_{0}} \frac{\partial w_{s}}{\partial \theta}\right)+\left(\frac{z \cos \varphi}{R_{0}}\right) \theta_{\varphi} \\
e_{\varphi \theta}=\frac{1}{R_{0}} \frac{\partial u_{s}}{\partial \theta}+\left(\frac{1}{R_{\varphi}}\right) \frac{\partial v_{s}}{\partial \varphi}-\left(\frac{\cos \varphi}{R_{0}}\right) v_{s}+\frac{2 z}{R_{0}} \frac{\partial \theta_{\varphi}}{\partial \theta}
\end{array}\right.
$$

By assuming that the dynamic external excitation is harmonic and expanding each variable function $f(\varphi, \theta, t)$ with the Fourier series along the circumferential direction, one obtains

$$
f(\varphi, \theta, t)=\left(\sum_{n} \widetilde{f}^{(n)}(\varphi) e^{j n \theta}\right) e^{j \omega t},
$$

where $\tilde{f}^{(n)}$ is the amplitude of the $n$th order component of $f$ in the frequency domain, $j=\sqrt{-1}$, and $\omega$ is the circular excitation frequency. In the following sections, the factors $e^{j \omega t}$ and $e^{j n \theta}$, and the superscript $n$ are omitted for convenience.

By combing equations (7) and (11) and applying a Fourier series expansion, the internal force-displacement relations of the solid frame are 


$$
\begin{aligned}
& \tilde{N}_{\theta}=\int_{-h / 2}^{h / 2} \tilde{\sigma}_{\theta} \mathrm{d} z=\left(\frac{\overline{\bar{A}}}{R_{\varphi}}\right) h \frac{\mathrm{d} \widetilde{u}_{s}}{\mathrm{~d} \varphi}+\left(\frac{\overline{\bar{N}} n j}{R_{0}}\right) h \widetilde{v}_{s}+\left(\frac{\overline{\bar{N}} \cos \varphi}{R_{0}}\right) h \widetilde{u}_{s}+\left[\left(\frac{\overline{\bar{N}} \sin \varphi}{R_{0}}\right)+\left(\frac{\overline{\bar{A}}}{R_{\varphi}}\right)\right] h \widetilde{w}_{s}+\overline{\bar{B}} h \widetilde{P}, \\
& \tilde{N}_{\varphi \theta}=\tilde{N}_{\theta \varphi}=\int_{-h / 2}^{h / 2} \tilde{\tau}_{\varphi \theta} \mathrm{d} z=\left(\frac{N h n j}{R_{0}}\right) \widetilde{u}_{s}+\left(\frac{N h}{R_{\varphi}}\right) \frac{\mathrm{d} \widetilde{v}_{s}}{\mathrm{~d} \varphi}-\left(\frac{N h \cos \varphi}{R_{0}}\right) \widetilde{v}_{s}, \\
& \tilde{M}_{\varphi}=\int_{-h / 2}^{h / 2} \widetilde{\sigma}_{\varphi} z \mathrm{~d} z=\left(\frac{h^{3}}{12}\right)\left(\frac{\overline{\bar{N}}}{R_{\varphi}}\right) \frac{\mathrm{d} \tilde{\theta}_{\varphi}}{\mathrm{d} \varphi}+\left(\frac{h^{3}}{12}\right)\left(\frac{\overline{\bar{A}} n j \sin \varphi}{R_{0}^{2}}\right) \widetilde{v}_{s}+\left(\frac{h^{3}}{12}\right)\left(\frac{\overline{\bar{A}} n^{2}}{R_{0}^{2}}\right) \widetilde{w}_{s}+\frac{h^{3}}{12}\left(\frac{\overline{\bar{A}} \cos \varphi}{R_{0}}\right) \tilde{\theta}_{\varphi}+\left(\frac{h^{3}}{12}\right) \overline{\bar{B}} \tilde{M}_{p}, \\
& \tilde{M}_{\theta}=\int_{-h / 2}^{h / 2} \widetilde{\sigma}_{\theta} z \mathrm{~d} z=\left(\frac{h^{3}}{12}\right)\left(\frac{\overline{\bar{N}} n j \sin \varphi}{R_{0}^{2}}\right) \widetilde{v}_{s}+\left(\frac{h^{3}}{12}\right)\left(\frac{\overline{\bar{N}} n^{2}}{R_{0}^{2}}\right) \widetilde{w}_{s}+\left(\frac{h^{3}}{12}\right)\left(\frac{\overline{\bar{A}}}{R_{\varphi}}\right) \frac{\mathrm{d} \tilde{\theta}_{\varphi}}{\mathrm{d} \varphi}+\left(\frac{h^{3}}{12}\right)\left(\frac{\overline{\bar{N}} \cos \varphi}{R_{0}}\right) \tilde{\theta}_{\varphi}+\left(\frac{h^{3}}{12}\right) \overline{\bar{B}} \tilde{M}_{p}, \\
& \tilde{M}_{\varphi \theta}=M_{\theta \varphi}=\int_{-h / 2}^{h / 2} \tilde{\tau}_{\varphi \theta} z \mathrm{~d} z=\left(\frac{h^{3}}{12}\right)\left(\frac{2 N n j}{R_{0}}\right) \tilde{\theta}_{\varphi} .
\end{aligned}
$$

In equation (13), the following midplane variables are $\sigma_{i j, j}-b\left(\dot{u}_{i}-\dot{U}_{i}\right)+F_{i}=\rho_{11} \ddot{u}_{i}+\rho_{12} \ddot{U}_{i}, \quad(i, j=1,2,3)$, introduced: $\widetilde{u}_{s}=(1 / h) \int_{-h / 2}^{h / 2} \widetilde{u}_{s} \mathrm{~d} z, \widetilde{v}_{s}=(1 / h) \int_{-h / 2}^{h / 2} \widetilde{v}_{s} \mathrm{~d} z, \widetilde{w}_{s}=$ $(1 / h) \int_{-h / 2}^{h / 2} \widetilde{w}_{s} \mathrm{~d} z, \widetilde{p}=(1 / h) \int_{-h / 2}^{h / 2} \phi \widetilde{p} \mathrm{~d} z, \tilde{M}_{p}=\left(12 / h^{3}\right) \int_{-h / 2}^{h / 2}$ $z \phi \widetilde{p} \mathrm{~d} z$, where $h$ is the thickness of the shell; $\tilde{N}_{\varphi}$ and $\tilde{N}_{\theta}$ are the component amplitudes of the axial force per unit length in the $\varphi$ and $\theta$ directions, respectively; $\widetilde{N}_{\varphi \theta}$ is the amplitude of the shear force per unit length; $\widetilde{M}_{\varphi}$ and $\widetilde{M}_{\theta}$ are the amplitudes of the bending moment per unit length in the $\varphi$ and $\theta$ directions, respectively; and $\tilde{M}_{\varphi \theta}$ is the amplitude of the torque per unit length.

According to the Biot theory, the dynamic equations of the solid frame are $[1,2]$

where the dots indicate differentiation with respect to time. $F_{i}(i=1,2,3)$ are the body forces per unit volume. $U_{i}$ is the fluid midplane displacement. $\rho_{11}=(1-\phi) \rho_{s}+\phi \rho_{f}\left(\alpha_{\infty}-1\right)$ and $\rho_{12}=\phi \rho_{f}\left(\alpha_{\infty}-1\right)$ are the mass density coefficients related to the frame and fluid mass densities, respectively. $\rho_{s}$ and $\rho_{f}$ are the frame mass density and fluid mass density, respectively. $\alpha_{\infty}$ is the tortuosity. $b=\left(v \phi^{2} / q\right)$ is a coefficient related to the permeability $q$, and $v$ is the fluid viscosity.

Based on thin shell theory and considering equation (14), the solid dynamic equations of the fluid-saturated porous rotational shell can be derived in the frequency domain as

$$
\begin{array}{r}
R_{\varphi} \cos \varphi \widetilde{N}_{\varphi}+R_{0} \frac{\partial \widetilde{N}_{\varphi}}{\partial \varphi}+R_{\varphi} \frac{\partial \widetilde{N}_{\theta \varphi}}{\partial \theta}-R_{\varphi} \cos \varphi \widetilde{N}_{\theta}+R_{0} \widetilde{Q}_{\varphi}+R_{\varphi} R_{0} \widetilde{q}_{\varphi}-R_{\varphi} R_{0} h\left(\Omega_{11} \widetilde{u}_{s}-\Omega_{12} \widetilde{u}_{f}\right)=0, \\
R_{\varphi} \frac{\partial \widetilde{N}_{\theta}}{\partial \theta}+2 R_{\varphi} \cos \varphi \widetilde{N}_{\varphi \theta}+R_{0} \frac{\partial \widetilde{N}_{\varphi \theta}}{\partial \varphi}+R_{\varphi} R_{0} \frac{\widetilde{Q}_{\theta}}{R_{\theta}}+R_{\varphi} R_{0} \widetilde{q}_{\theta}-R_{\varphi} R_{0} h\left(\Omega_{11} \widetilde{v}_{s}-\Omega_{12} \widetilde{v}_{f}\right)=0, \\
R_{\varphi} \cos \varphi \widetilde{Q}_{\varphi}+R_{0} \frac{\partial \widetilde{Q}_{\varphi}}{\partial \varphi}+R_{\varphi} \frac{\partial \widetilde{Q}_{\theta}}{\partial \theta}-R_{\varphi} R_{0} \frac{\widetilde{N}_{\varphi}}{R_{\varphi}}-R_{\varphi} R_{0} \frac{\widetilde{N}_{\theta}}{R_{\theta}}+R_{\varphi} R_{0} \widetilde{q}_{z}-R_{\varphi} R_{0} h\left(\Omega_{11} \widetilde{w}_{s}-\Omega_{12} \widetilde{w}_{f}\right)=0, \\
R_{\varphi} \frac{\partial \widetilde{M}_{\theta}}{\partial \theta}+R_{\varphi} \cos \varphi \widetilde{M}_{\varphi \theta}+R_{0} \frac{\partial \widetilde{M}_{\varphi \theta}}{\partial \varphi}+R_{\varphi} \cos \varphi \widetilde{M}_{\theta \varphi}-R_{\varphi} R_{0} \widetilde{Q}_{\theta}=0, \\
R_{\varphi} \cos \varphi \widetilde{M}_{\varphi}+R_{0} \frac{\partial \widetilde{M}_{\varphi}}{\partial \varphi}+R_{\varphi} \frac{\partial \widetilde{M}_{\beta \varphi}}{\partial \theta}-R_{\varphi} \cos \varphi \widetilde{M}_{\theta}-R_{\varphi} R_{0} \widetilde{Q}_{\varphi}=0,
\end{array}
$$

where $\Omega_{11}=b \omega j-\rho_{11} \omega^{2}$ and $\Omega_{12}=\rho_{12} \omega^{2} \widetilde{u}_{f}+b \omega j$ are frequency parameters. $\widetilde{u}_{f}=\widetilde{U}_{1}, \widetilde{v}_{f}=\widetilde{U}_{2}$ and $\widetilde{W}_{f}=\widetilde{U}_{3}$ are the amplitudes of the fluid midplane displacements. $\tilde{q}_{i}(i=\varphi, \theta, z)$ is the amplitudes of external loads. $Q_{\varphi}$ and $Q_{\theta}$ are the amplitudes of the shear forces per unit length in the $\varphi$ and $\theta$ directions, respectively. These forces can be represented by Kelvin-Kirchhoff equivalent shear forces as follows: 


$$
\begin{gathered}
\widetilde{S}_{\varphi}=\widetilde{N}_{\varphi \theta}+\frac{\sin \varphi}{R_{0}} \widetilde{M}_{\varphi \theta}, \\
\widetilde{V}_{\varphi}=\widetilde{Q}_{\varphi}+\left(\frac{n j}{R_{0}}\right) \tilde{M}_{\varphi \theta} .
\end{gathered}
$$

2.3. Governing Equations of the Fluid. By adopting the Biot theory, the dynamic equations of the fluid are $[1,2]$

$$
(-\phi p)_{, i}+b\left(\dot{u}_{i}-\dot{U}_{i}\right)+X_{i}=\rho_{21} \ddot{u}_{i}+\rho_{22} \ddot{U}_{i},
$$

where $X_{i}$ is the body force vector per unit volume. $\rho_{22}=$ $\phi \rho_{f}+\phi \rho_{f}\left(\alpha_{\infty}-1\right)$ and $\rho_{21}=\rho_{12}$ are the mass density coefficients related to the frame and fluid mass densities, respectively.

Thus, based on thin shell theory, the fluid dynamic equations of the fluid-saturated porous rotational shell are written in the frequency domain as

$$
\begin{array}{r}
-\frac{1}{R_{\varphi}} \frac{\mathrm{d}(\phi \tilde{p})}{\mathrm{d} \varphi}=-\Omega_{21} \widetilde{\widehat{u}}_{s}+\Omega_{22} \widetilde{\hat{u}}_{f}, \\
-\left(\frac{n j}{R_{0}}\right)(\phi \widetilde{p})=-\Omega_{21} \widetilde{\widetilde{v}}_{s}+\Omega_{22} \widetilde{\widetilde{v}}_{f}, \\
-\frac{\mathrm{d}(\phi \tilde{p})}{\mathrm{d} z}=-\Omega_{21} \widetilde{\widetilde{w}}_{s}+\Omega_{22} \widetilde{\widetilde{w}}_{f},
\end{array}
$$

where $\widetilde{\widehat{u}}, \widetilde{\widehat{v}}_{f}$, and $\widetilde{\widehat{w}}_{f}$ are the amplitudes of the fluid displacements at arbitrary point of the porous shell. As well, $\Omega_{21}=b \omega j+\rho_{21} \omega^{2}$ and $\Omega_{22}=b \omega j-\rho_{22} \omega^{2}$ are the frequency parameters.
The amplitudes of the fluid displacements can be determined from equation (18) as

$$
\begin{aligned}
& \widetilde{\widehat{u}}_{f}=\left(\frac{\Omega_{21}}{\Omega_{22}}\right) \widetilde{\widehat{u}}_{s}-\left(\frac{1}{R_{\varphi} \Omega_{22}}\right) \frac{\mathrm{d}(\phi \tilde{p})}{\mathrm{d} \varphi}, \\
& \widetilde{\widehat{v}}_{f}=\left(\frac{\Omega_{21}}{\Omega_{22}}\right) \widetilde{\widehat{v}}_{s}-\left(\frac{n j}{R_{0} \Omega_{22}}\right)(\phi \tilde{p}), \\
& \widetilde{\widetilde{w}}_{f}=\left(\frac{\Omega_{21}}{\Omega_{22}}\right) \widetilde{\widehat{w}}_{s}-\left(\frac{1}{\Omega_{22}}\right) \frac{\mathrm{d}(\phi \tilde{p})}{\mathrm{d} z} .
\end{aligned}
$$

The fluid strain-displacement relations of the fluid-saturated porous rotational shell are

$$
\begin{aligned}
& \widetilde{\varepsilon}_{\varphi}=\frac{1}{R_{\varphi}}\left(\frac{\partial \widetilde{\tilde{u}}_{f}}{\partial \varphi}+\widetilde{\widetilde{w}}_{f}\right), \\
& \widetilde{\varepsilon}_{\theta}=\frac{1}{R_{0}}\left(\widetilde{\widehat{u}}_{f} \cos \varphi+n j \widetilde{\widehat{v}}_{f}+\sin \varphi \widetilde{\widehat{w}}_{f}\right), \\
& \widetilde{\varepsilon}_{z}=\frac{\partial \widetilde{\widehat{w}}_{f}}{\partial z} \approx 0 .
\end{aligned}
$$

Inserting the third formula of equation (19) into the third formula of equation (20), it can be shown that $\left(\mathrm{d}^{2}(\phi \tilde{p}) / \mathrm{d} z^{2}\right)=0$ since the derivative $\left(\mathrm{d} \widetilde{\widehat{w}}_{s} / \mathrm{d} z\right)$ is nearly equal to zero for a thin shell. Therefore, it is reasonable to assume that the fluid pressure, $\widetilde{p}$, within a thin shell, is linearly distributed along $z$ direction.

Combining equations (8) and (20), the fluid constitutive equations of a porous rotational shell are derived in the frequency domain as

$$
\left(\frac{1}{R_{\varphi}}\right) \frac{\mathrm{d} \widetilde{\hat{u}}_{f}}{\mathrm{~d} \varphi}+\left(\frac{n j}{R_{0}}\right) \widetilde{\hat{v}}_{f}+\left(\frac{\cos \varphi}{R_{0}}\right) \widetilde{\widetilde{u}}_{f}+\left(\frac{\sin \varphi}{R_{0}}+\frac{1}{R_{\varphi}}\right) \widetilde{\widetilde{w}}_{f}=\overline{\bar{A}}^{\prime} \phi p+\overline{\bar{B}}^{\prime}\left(e_{\varphi}+e_{\theta}\right) .
$$

Substituting equation (11) into equation (21), integrating over the shell thickness with the aid of equation (9), and assuming the fluid pressure in linearly distributed along the $z$-direction, one can achieve

$$
\begin{aligned}
& -R_{\varphi}\left(\Omega_{21}-\Omega_{22} \overline{\bar{B}}^{\prime}\right) \frac{\mathrm{d} \widetilde{u}_{s}}{\mathrm{~d} \varphi}+\left[\left(\frac{R_{\varphi} \cos \varphi}{R_{0}}\right)-\left(\frac{R_{\varphi}^{\prime}}{R_{\varphi}}\right)\right] \frac{\mathrm{d} P}{\mathrm{~d} \varphi}+\frac{\mathrm{d}^{2} P}{\mathrm{~d} \varphi^{2}} \\
= & \left(\frac{R_{\varphi}^{2} \cos \varphi}{R_{0}}\right)\left(\Omega_{21}-\Omega_{22} \overline{\bar{B}}^{\prime}\right) \widetilde{u}_{s}+\left(\frac{R_{\varphi}^{2} n j}{R_{0}}\right)\left(\Omega_{21}-\Omega_{22} \overline{\bar{B}}^{\prime}\right) \widetilde{v}_{s}+R_{\varphi}^{2}\left(\frac{\sin \varphi}{R_{0}}+\frac{1}{R_{\varphi}}\right)\left(\Omega_{21}-\Omega_{22} \overline{\bar{B}}^{\prime}\right) \widetilde{w}_{s} \\
& -R_{\varphi}^{2}\left(\frac{n^{2}}{R_{0}^{2}}+\Omega_{22} \overline{\bar{A}}^{\prime}\right) \widetilde{P}-\left(\frac{\sin \varphi}{R_{0}}+\frac{1}{R_{\varphi}}\right)\left(\frac{R_{\varphi}^{2}}{h}\right) \phi\left(\widetilde{p}_{1}-\widetilde{p}_{2}\right),
\end{aligned}
$$


where $R_{\varphi}^{\prime}=\left(\partial R_{\varphi} / \partial \varphi\right)$, and $\widetilde{p}_{1}$ and $\widetilde{p}_{2}$ are the amplitudes of the pressure on the surface of the shell.
Similarly, multiplying equation (21) by $z$, and integrating over the thickness of the shell with the aid of equation (9), one can obtain

$$
\begin{aligned}
& -\left(\frac{1}{R_{\varphi}^{2}}\right) \frac{\mathrm{d}^{2} \widetilde{M}_{P}}{\mathrm{~d} \varphi^{2}}-\left[\left(\frac{n^{2}}{R_{0}^{2}}\right)+\Omega_{22} \overline{\bar{A}}^{\prime}\right] M_{P}+\left(\frac{R_{\varphi}^{\prime}}{R_{\varphi}^{3}}\right) \frac{\mathrm{d} \widetilde{M}_{P}}{\mathrm{~d} \varphi}+\left(\Omega_{21}-\Omega_{22} \overline{\bar{B}}^{\prime}\right)\left(\frac{n j \sin \varphi}{R_{0}^{2}}\right) \widetilde{v}_{s} \\
& -\left(\Omega_{21}-\Omega_{22} \overline{\bar{B}}^{\prime}\right)\left(\frac{n^{2}}{R_{0}^{2}}\right) \widetilde{w}_{s}+\left(\Omega_{21}-\Omega_{22} \overline{\bar{B}}^{\prime}\right)\left(\frac{1}{R_{\varphi}}\right) \frac{\mathrm{d} \theta_{\varphi}}{\mathrm{d} \varphi}+\left[\Omega_{21}-\Omega_{22} \overline{\bar{B}}^{\prime}\right]\left(\frac{\cos \varphi}{R_{0}}\right) \theta_{\varphi}=0 .
\end{aligned}
$$

Equations (22) and (23) are the governing equations of the fluid in the fluid-statured porous rotational shell. It should be emphasized that, in the above derivation, there are not assumptions for the displacements. Meanwhile, the coupling interaction between the phases are considered. The absence of displacement simplifications and consideration of fluid-solid coupling is an advantage of the present method.

2.4. The Integrated Governing Equations of a Porous Rotational Shell Saturated in Fluids. By introducing dimensionless variables, the physical variables can be expressed as

$$
\begin{aligned}
& \widetilde{u}_{s}=L \bar{u}_{s}, \\
& \widetilde{v}_{s}=L \bar{v}_{s} \text {, } \\
& \widetilde{w}=L \bar{w}_{s}, \\
& \tilde{\theta}_{\varphi}=\bar{\theta}_{\varphi}, \\
& \tilde{N}_{\varphi}=(h \overline{\bar{N}}) \bar{N}_{\varphi} \text {, } \\
& \widetilde{S}_{\varphi}=(h \overline{\bar{N}}) \bar{S}_{\varphi}, \\
& \widetilde{V}_{\varphi}=(h \overline{\bar{N}}) \bar{V}_{\varphi} \text {, } \\
& \tilde{M}_{\varphi}=\left(\frac{\overline{\bar{N}} h^{3}}{12 L}\right) \bar{M}_{\varphi}, \\
& \widetilde{P}=\frac{\overline{\bar{N}}}{\overline{\bar{B}}} \bar{P}, \\
& \tilde{M}_{p}=\left(\frac{\overline{\bar{N}}}{\overline{\bar{B}} L}\right) \bar{M}_{p}, \\
& \widetilde{q}_{\varphi}=\left(\frac{h \overline{\bar{N}}}{L}\right) \bar{q}_{\varphi}, \\
& \tilde{q}_{\theta}=\left(\frac{h \overline{\bar{N}}}{L}\right) \bar{q}_{\theta}, \\
& \widetilde{q}_{z}=\left(\frac{h \overline{\bar{N}}}{L}\right) \bar{q}_{z},
\end{aligned}
$$

where $L$ is a length for nondimensionalization.
Combining the solid governing equations (10), (13), (15), and (16), and the fluid governing equations (22) and (23), the integrated governing equations of a porous rotational shell can be obtained after the elimination of some dependent variables. After implementing the dimensionless procedure using equation (24), the integrated governing equations can be expressed as the following first-order differential matrix equation:

$$
\mathbf{A} \frac{\mathrm{d} \mathbf{Z}}{\mathrm{d} \varphi}=\mathbf{B Z}+\mathbf{F}
$$

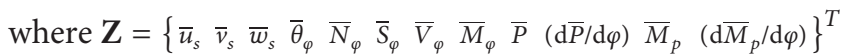
is the integrated state vector, $\mathbf{A}$ and $\mathbf{B}$ are coefficient matrixes, and $\mathbf{F}$ is the integrated load vector. The nonzero components are listed in Appendix.

Equation (25) can be solved effectively by the extended homogeneous capacity precision integration method [17]. The relationship between the start point and end point is written as

$$
\mathbf{Z}_{e}=\mathbf{T} \mathbf{Z}_{s}
$$

where $\mathbf{Z}_{e}$ and $\mathbf{Z}_{s}$ are the state vector of the start point and end point, respectively, and $T$ is the transfer matrix.

In general, there are six known boundary conditions and six unknown boundary conditions at each boundary. Taking the fixed end as an example, its known boundary conditions are $\bar{u}_{s}, \bar{v}_{s}, \bar{w}_{s}, \bar{\theta}_{\varphi}, \bar{P}, \bar{M}_{p}$, denoted as $\mathbf{Z}_{k}$, while its unknown boundary conditions can be denoted as $\mathbf{Z}_{u}$. Applying the boundary conditions to equation (26), the unknown boundary conditions $\mathbf{Z}_{u}$ are determined with some rearrangement of transfer matrix $T$. Combining the 12 boundary conditions of the starting point and precision integration method, the integrated state vector at any point of the shell is obtained based on equation (25).

\section{Results and Discussion}

3.1. Comparison with the Experiment Result. To verify the present model, a vibration experiment of a cantilevered cylindrical shell is performed. The experiment is degenerated from the porous rotational shell when $R_{\varphi} \longrightarrow \infty$, $R_{0}=R, \varphi=(\pi / 2)$. The shell is made of the foamed aluminium, and the geometric and physical parameters are listed in Table 1. The experimental setup is shown in Figure 2. In this case, a unit harmonic excitation is loaded 
TABle 1: Parameters of the porous cylinder shell.

\begin{tabular}{lcc}
\hline Properties & Description & Value \\
\hline$L$ & Length & $500 \mathrm{~mm}$ \\
$R$ & Radius & $100 \mathrm{~mm}$ \\
$H$ & Thickness & $10 \mathrm{~mm}$ \\
$\rho_{s}$ & Solid density & $2700 \mathrm{~kg} / \mathrm{m}^{3}$ \\
$\rho_{f}$ & Fluid density & $1.213 \mathrm{~kg} / \mathrm{m}^{3}$ \\
$\mu$ & Poisson's ratio & 0.3 \\
$E$ & Young's modulus & $0.48 \mathrm{GPa}$ \\
$\phi$ & Porosity & 0.74 \\
$\alpha_{\infty}$ & Tortuosity & 1.2 \\
$q$ & Permeability & $2.7 \times 10^{-10} \mathrm{~m}^{2}$ \\
\hline
\end{tabular}

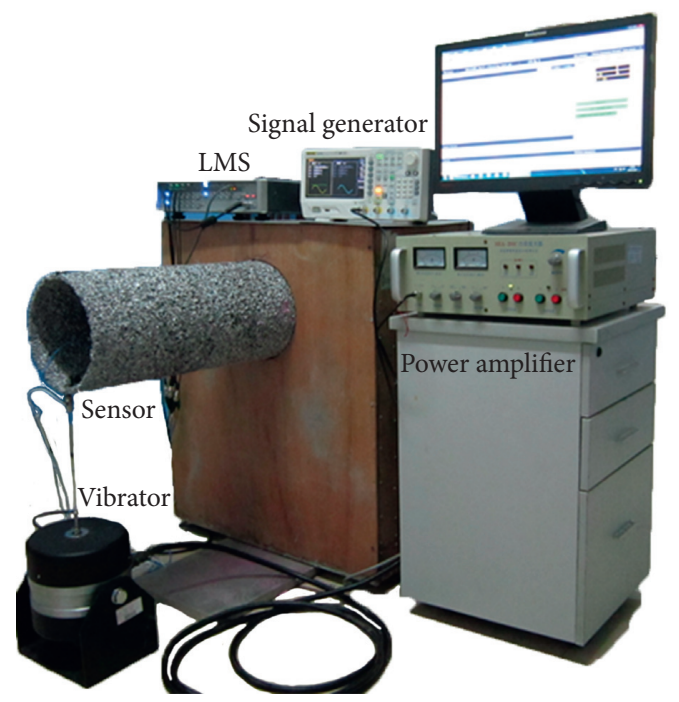

FIGURE 2: Vibration experiment of a porous cylinder shell.

at the bottom of the free end $(\theta=0)$. The deflections of the bottom and top points at the free end are both measured. The frequency response function is then given by $\mathrm{FRF}=\log _{10}(|\bar{w}|)$, in which $\bar{w}$ is the dimensionless displacement amplitude of the free end. The experimental results compared with the numerical ones obtained by the present method are shown in Figure 3. The results given by the two method are very similar in most frequency range, especially the values of the natural frequencies. The relative errors of the first- and second-order natural frequencies are both less than $3 \%$. It should be noted that, due to the singularity of the theoretical model and insufficient consideration of the damping in the experiment, the vibration amplitude at the natural frequency is far from the experimental value.

3.2. Comparison with Equivalent Models. Among all the porous material models with saturated fluid, equivalent models are the most widely used, such as the effective medium method and the equivalent density fluid method. The former method is applied to the porous material in case of a rigid solid frame. It assumes that the microelements of the porous materials can be seen as an effective elastic

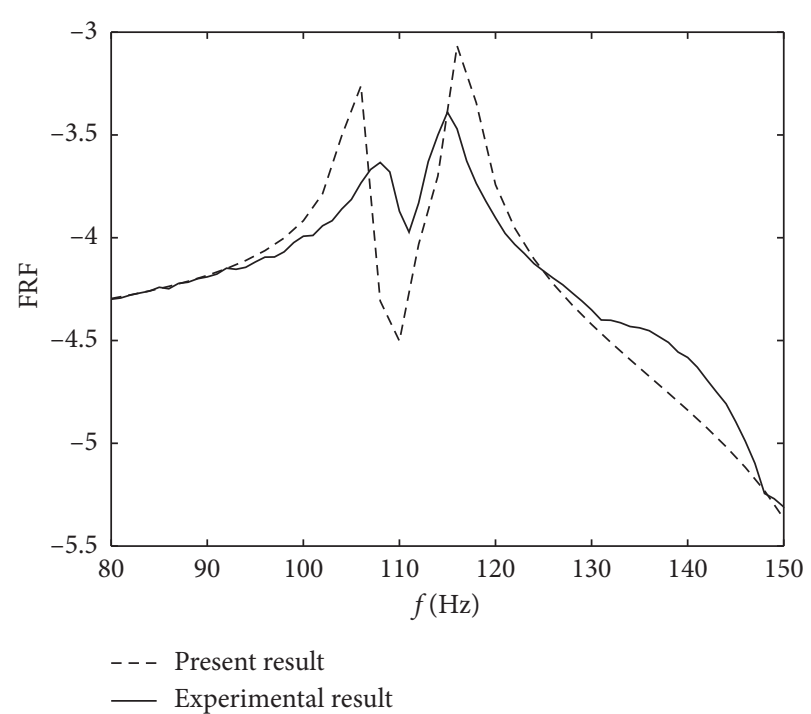

FIgURE 3: Comparison between experimental and present result.

element [18]. The latter method is used for porous material in the case of a limp solid frame. Here, it is assumed that the frame is almost as soft as a fluid, and the microelements of the porous material can be treated as an effective limp element. Both models describe the porous mediums' mechanical behaviours by using an equivalent mass density and ignore the solid-fluid coupling.

In this section, two cases are considered for two different kinds of rotational shells using the effective medium method and the equivalent density fluid method. The numerical results are compared with the ones obtained by the present method.

The effective medium method combined with FEM software is used to analyze a foamed aluminium cantilever shell and compared with the proposed method. For the calculation, the shell has a major axis $a=21 \mathrm{~mm}$, minor axis $b=105 \mathrm{~mm}$, starting angle $\varphi=(\pi / 6)$, end angle $\varphi=((5 \pi) / 6)$, and layer thicknesses $h=210 \mathrm{~mm}$. The material parameters are listed in Table 2. Applying the equivalent elastic modulus and the equivalent density in FEM software, the comparison of the results for the natural frequencies is listed in Table 3 with $m$ denoting the half wave numbers of the meridian direction and $n$ denoting that of the circumferential direction. Table 3 indicates that the proposed method and the equivalent medium method have good agreement with each other.

For the limp framed porous shell, the effective density fluid model is used. In this case, the solid-fluid coupling is negligible $\left(u=U, \rho_{12}=0\right)$ [19], and the porous material is described by an effective bulk modulus $K_{\text {eff }}$ and an effective density $\rho_{\text {eff }}$ :

$$
\begin{aligned}
K_{\text {eff }} & =\left(\frac{1-\phi}{K_{s}}+\frac{\phi}{K_{f}}\right)^{-1}, \\
\rho_{\text {eff }} & =\frac{\rho \tilde{\rho}-\rho_{f}^{2}}{\tilde{\rho}+\rho-2 \rho_{f}},
\end{aligned}
$$


TABle 2: Parameters of the rigid framed rotational shell.

\begin{tabular}{lcccccc}
\hline$\rho_{s}$ & $\rho_{f}$ & $\mu$ & $E_{s}(\mathrm{GPa})$ & $\phi$ & $q$ & $\alpha_{\infty}$ \\
\hline $2700 \mathrm{~kg} / \mathrm{m}^{3}$ & $1.213 \mathrm{~kg} / \mathrm{m}^{3}$ & 0.3 & 6.9 & 0.3 & $2.7 \times 10^{-10} \mathrm{~m}^{2}$ \\
\hline
\end{tabular}

TABle 3: Comparison of natural frequency.

\begin{tabular}{lcc}
\hline Order $(m, n)$ & Present method $(\mathrm{Hz})$ & FEM $(\mathrm{Hz})$ \\
\hline$(1,1)$ & 76.37 & 74.72 \\
$(1,2)$ & 181.93 & 180.92 \\
$(1,3)$ & 233.78 & 234.92 \\
$(1,4)$ & 369.00 & 375.60 \\
$(1,5)$ & 610.50 & 621.37 \\
\hline
\end{tabular}

TABLe 4: Parameters of the limp frame porous rotational shell.

\begin{tabular}{lcccccccc}
\hline$\rho_{s}$ & $\rho_{f}$ & $\mu$ & $E(\mathrm{GPa})$ & $\phi$ & $q$ & $\alpha_{\infty}$ & $Y$ & $F$ \\
\hline $1136 \mathrm{~kg} / \mathrm{m}^{3}$ & $1.293 \mathrm{~kg} / \mathrm{m}^{3}$ & 0.35 & 0.21 & 0.69 & $1 \times 10^{-8} \mathrm{~m}^{2}$ & 1.03 & $0.00105 \mathrm{~kg} / \mathrm{m} \cdot \mathrm{s}$ & 1.0 \\
\hline
\end{tabular}

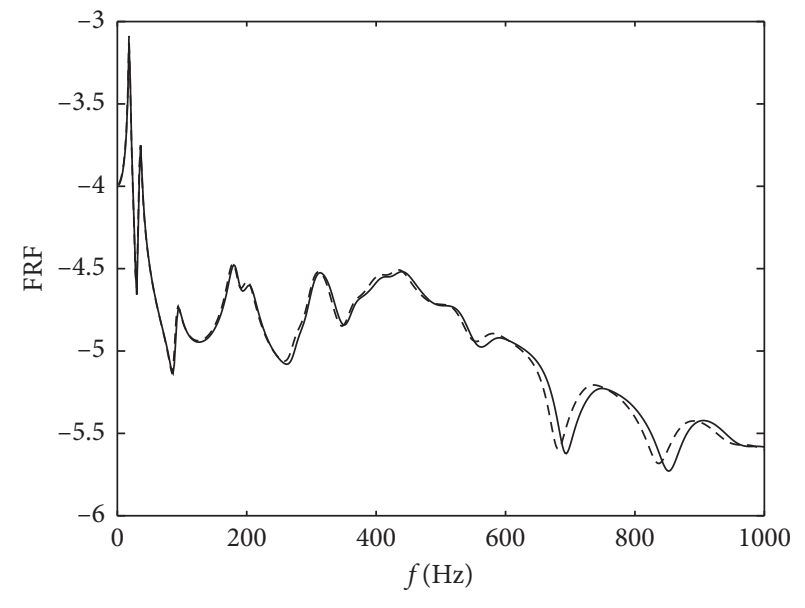

_ Equivalent fluid model

- - - Present model

FIgURE 4: Comparison between the effective density fluid method and the present method.

where $\tilde{\rho}=\left(\left(\alpha_{\infty} \rho_{f}\right) / \phi\right)+((j F v) /(q \omega))$ and $\rho=\phi \rho_{f}+(1-$ $\phi) \rho_{s}$, and $K_{s}$ and $K_{f}$ are the bulk modulus of the solid and the pore fluid, respectively. $v$ is the dynamic viscosity coefficient of the fluid, and $F$ is also a coefficient of the fluid. For this calculation, a thin $Y$ foam rotational shell with major axis $a=260 \mathrm{~mm}$, minor axis $b=105 \mathrm{~mm}$, starting angle (free end) $\varphi=(\pi / 6)$, and end angle (fixed end) $\varphi=(\pi / 2)$ is considered and surrounded by water. The shell is excited by a unit harmonic excitation $F_{z}=1 e^{j \omega t} N$ at the free end $(\varphi=(\pi / 6), \theta=0)$, and $K_{s}=6.9 \mathrm{Gpa}$ and $K_{f}=2.395 \mathrm{Gpa}$. The parameters of the shell are listed in Table 4 . Figure 4 shows the frequency response curves given by the present method and the equivalent density fluid method.

By comparing the curves in Figure 4, we note that the two methods give very similar results at low frequencies,

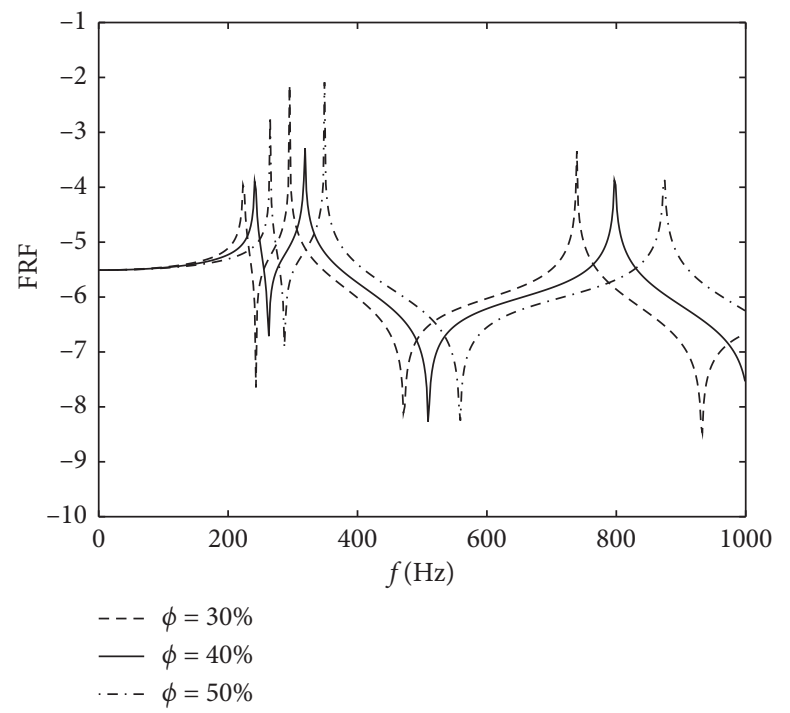

FIGURE 5: FRF curve for various porosities.

but differences in results arise at higher frequencies. This may be due to the fact that the present method has fully considered the interactions between the fluid and solid phases, while the equivalent density fluid method has ignored this effect, so this simplification is only valid at low frequencies. As the frequency increases, the interactions between the fluid and solid phases gradually increase and it can no longer be neglected at higher frequencies. Therefore, compared to the equivalent fluid density method, the present model can be applied over a wider frequency range.

3.3. The Effects of Parameters. In this section, the influence of material and structural parameters, for instance, porosity, 


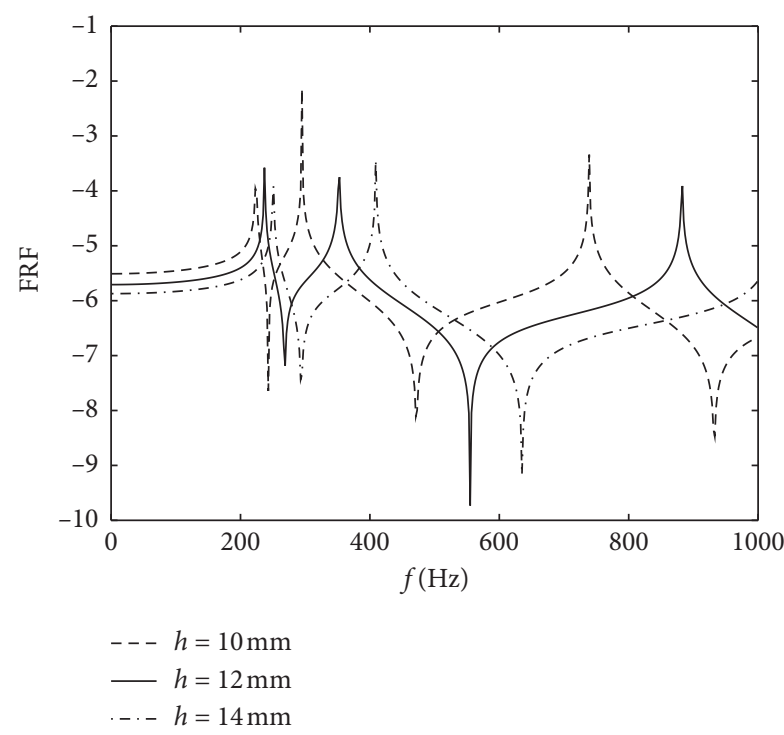

FIGURE 6: FRF curve for various thicknesses.

thickness, and ellipticity on structural vibration have been further studied. Letting the end angle $\varphi=(\pi / 2)$, a porous rotational shell, the same as the first case in Section 3.2, is considered with a unit harmonic excitation $F_{z}=1 e^{j \omega t} N$ excited at the free end. Figure 5 gives the amplitude frequency response curves with porosities of $0.3,0.4$, and 0.5 . We note from the figure that higher porosity results in higher resonant frequencies. However, higher porosity does not always damp better, especially in the low-frequency range. This observation may be explained by the fact that the increasing porosity leads to the reduction of equivalent mass and equivalent stiffness. The former will reduce the vibration, and the latter will increase it. The descent velocities of the two factors is different in different frequency ranges, so the variation of resonance peak with porosity is not uniform.

To demonstrate the effect of the thickness, different thicknesses $(10 \mathrm{~mm}, 12 \mathrm{~mm}$, and $14 \mathrm{~mm})$ are examined with the porosity of $30 \%$. The response curves are illustrated in Figure 6. It can be concluded from the figure that increasing the shell thickness leads to an increase in the resonant frequencies. However, increasing the thickness does not necessarily achieve better damping effect, e.g., the vibration at the first resonance peak. The interpretation of this behaviour is that although thicker structure can increase energy loss, it also can increase the vibration for the increases of the inertia.

For a thickness of $10 \mathrm{~mm}$, the influence of ellipticity on the vibrational performance of the shell is discussed. In the meridian plane, the geometric equation of the porous rotational shell is $(z / a)^{2}+(r / b)^{2}=1$. The effects of the ellipticity on the frequency response curves are shown in Figure 7 with various values of ellipticity $(a / b=2.0,2.5$, and 3.0). It is seen from the figure that the resonant frequencies decrease as the ellipticity is increased. As well, this phenomenon is more obvious in the low-frequency range.

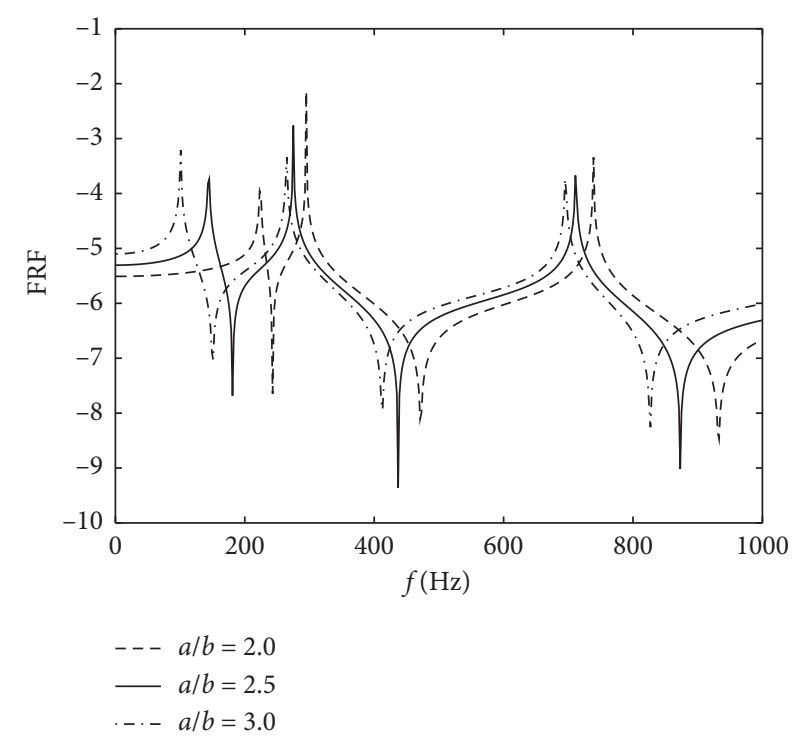

FIgURE 7: FRF curve for various ellipticities.

\section{Conclusions}

In this paper, a new theoretical model of thin porous rotational shells has been established based on the Biot theory and the classical elastic thin shell theory. The accuracy of the present model has been verified by comparing to experimental results and FEM results combined with effective medium method. In the low-frequency range, the proposed model agrees well with the above methods. However, in medium- and high-frequency ranges, the results do not agree well. This is certainly a consequence of the fact that the present model does not make any assumptions for the displacements and considers the interactions between the fluid and solid phases. These factors, usually neglected in the finite element method combined with the equivalent model, are more significant in medium and high frequencies. It indicates that the present model can be applied over a wide frequency range. The present investigation of the parameters of the porous rotational shell leads to the following conclusions: (i) increasing porosity and the thickness of the shell can increase in the resonant frequencies. (ii) The influence of porosity and thickness on the damping effect varies with the frequency. (iii) Increasing the ellipticity reduces the resonance amplitudes and frequencies. The effect of ellipticity in low frequency is more pronounced than that at medium and high frequencies.

\section{Data Availability}

The data used to support the findings of this study are included within the article, and there are not any restrictions on data access.

\section{Conflicts of Interest}

The authors declare no conflicts of interest. 


\section{Acknowledgments}

This work was supported by the National Natural Science Foundation of China (nos. 51665006, 51775121, and 11502056) and the Innovation Project of Guangxi University of Science and Technology Graduate Education (GKYC202001).

\section{Supplementary Materials}

The nonzero elements of the coefficient matrix and the nonzero elements of the load vector are shown in Appendix. (Supplementary Materials)

\section{References}

[1] M. A. Biot, "Theory of propagation of elastic waves in a fluidsaturated porous solid. I. low-frequency range," Journal of the Acoustical Society of America, vol. 2, no. 28, pp. 168-178, 1956.

[2] M. A. Biot, "Theory of propagation of elastic waves in a fluidsaturated porous solid. II. high-frequency range," Journal of the Acoustical Society of America, vol. 2, no. 28, pp. 179-191, 1956.

[3] O. C. Zienkiewicz, C. T. Chang, and P. Bettess, "Drained, undrained, consolidating, and dynamic behavior assumptions in soils: limits of validity," Geotechnique, vol. 4, no. 30, pp. 385-395, 1980.

[4] O. C. Zienkiewicz, "Basic formulation of static and dynamic behaviours of soil and other porous media," Journal of Applied Mathematics and Mechanics, vol. 4, no. 3, pp. 457-468, 1982.

[5] M. Cieszko and J. Kubik, "Bending vibration of a rectangular poroelastic plate," International Journal of Engineering Science, vol. 152, Article ID 103292, 2020.

[6] E. Manuel, S. Sohbi, and B. Bruno, "Theory of propagation of elastic waves in a fluid-saturated porous solid. I. low-frequency range," Comptes Rendus de I'Académie des Sciences, vol. 329, pp. 615-620, 2001.

[7] P. Leclaire and K. V. Horoshenkov, "Transverse vibrations of a thin rectangular porous plate saturated by a fluid," Journal of Sound and Vibration, vol. 1, no. 247, pp. 1-18, 2001.

[8] Y. Xiang, H. Jiang, and J. Lu, "Analyses of dynamic characteristics of a fluid-filled thin rectangular porous plate with various boundary conditions," Acta Mechanica Solida Sinica, vol. 30, no. 1, pp. 87-97, 2017.

[9] D. D. Theodorakopoulos and D. E. Beskos, "Flexural vibrations of poroelastic plates," Acta Mechanica, vol. 103, no. 1-4, pp. 191-203, 1994.

[10] S. Ahmed Shah, "Axially symmetric vibrations of fluid-filled poroelastic circular cylindrical shells," Journal of Sound and Vibration, vol. 318, no. 1-2, pp. 389-405, 2008.

[11] M. Julien, A. H. Mohamed, C. Jean-Daniel et al., "A mixed "Biot- Shell" analytical model for the prediction of sound transmission through a sandwich cylinder with a poroelastic core," Journal of Sound and Vibration, vol. 360, pp. 203-223, 2016.

[12] M. H. Jalaei and Ö. Civalek, "On dynamic instability of magnetically embedded viscoelastic porous FG nanobeam," International Journal of Engineering Science, vol. 143, pp. 14-32, 2019.

[13] M. Arefi, S. Firouzeh, E. Mohammad-Rezaei Bidgoli, and Ö. Civalek, "Analysis of porous micro-plates reinforced with FG-GNPs based on Reddy plate theory," Composite Structures, vol. 247, Article ID 112391, 2020.
[14] Y. J. Kang and J. S. Bolton, "Finite element modeling of isotropic elastic porous materials coupled with acoustical finite elements," Journal of the Acoustical Society of America, vol. 1, no. 98, pp. 635-643, 1995.

[15] C. Xu, J. Song, X. Du, and Z. Zhong, “A completely explicit finite element method for solving dynamic $u$ - $p$ equations of fluid-saturated porous media," Soil Dynamics and Earthquake Engineering, vol. 97, pp. 364-376, 2017.

[16] S. Boily and F. Charron, "The vibroacoustic response of a cylindrical shell structure with viscoelastic and poroelastic materials," Applied Acoustics, vol. 58, no. 2, pp. 131-152, 1999.

[17] Q. Ni, Y. Xiang, Y. Huang, and J. Lu, "Modeling and dynamics analysis of shells of revolution by partially active constrained layer damping treatment," Acta Mechanica Solida Sinica, vol. 26, no. 5, pp. 468-479, 2013.

[18] B. Qin, B. Liang, Z. M. Zhu et al., "Effective medium method of slightly compressible elastic media permeated with air-filled bubbles," Frontiers of Physics, vol. 4, no. 1, pp. 500-505, 2006.

[19] Z. J. Liu, T. D. Xia, R. Huang et al., "Comparison and discussion for Biot theory and modified Biot one," Journal of Vibration and Shock (in Chinese), vol. 34, no. 4, pp. 148-150, 2015. 